\title{
Intraoperative intravenous lidocaine exerts a protective effect on cell-mediated immunity in patients undergoing radical hysterectomy
}

\author{
HUAN-LIANG WANG ${ }^{1}$, HONG-DAN YAN ${ }^{1}$, YA-YANG LIU ${ }^{1,2}$, BAO-ZHU SUN ${ }^{1}$, \\ RUI HUANG ${ }^{1}$, XIAO-SHUANG WANG ${ }^{3}$ and WEI-FU LEI ${ }^{1}$
}

\author{
${ }^{1}$ Department of Anesthesiology, Qilu Hospital of Shandong University, Jinan, Shandong 250012; \\ ${ }^{2}$ Department of Anesthesiology, Hospital Affiliated to Shandong University of Traditional Chinese Medicine, \\ Jinan, Shandong 250000; ${ }^{3}$ College of Pharmacy, Shandong University of Traditional Chinese Medicine, \\ Jinan, Shandong 250000, P.R. China
}

Received October 26, 2014; Accepted July 29, 2015

DOI: $10.3892 / \mathrm{mmr} .2015 .4235$

\begin{abstract}
Surgical procedures cause a decrease in lymphocyte proliferation rate, an increase in apoptosis and shifts the balance of T-helper (Th)1/Th2 cells towards anti-cell-mediated immunity (CMI) Th2 dominance, which is relevant to the immunosuppressive effects of CMI, postoperative septic complications and the formation of tumor metastasis. Previous studies have revealed that lidocaine exhibits antibacterial actions; regulating inflammatory responses, reducing postoperative pain and affecting the duration spent in hospital. Thus, the present study hypothesized that lidocaine may exert a protective effect on the CMI of patients undergoing surgery for the removal of a primary tumor. A total of 30 adult female patients diagnosed with cervical cancer were recruited to the present study and were randomized into two groups. The lidocaine group received an intravenous bolus dose of $1.5 \mathrm{mg} / \mathrm{kg}$ lidocaine, followed by continuous infusion at $1.5 \mathrm{mg} / \mathrm{kg} / \mathrm{h}$ until discharge from the operating room. The control group received the same volume of normal saline. A $10 \mathrm{ml}$ sample of venous blood was drawn, and the lymphocytes were isolated using Ficoll-paque 1 day prior to surgery, at discharge from the operating room and $48 \mathrm{~h}$ post-surgery. The proliferation rate of the lymphocytes was assessed using a Cell Counting Kit-8 assay and was found to be higher in the lidocaine group. The early apoptosis of lymphocytes was attenuated following lidocaine treatment at $48 \mathrm{~h}$ post-surgery, as detected using flow cytometry with Annexin V-fluorescein isothiocyanate/propidium iodide staining. The level of interferon (IFN) $-\gamma$ in the
\end{abstract}

Correspondence to: Professor Wei-Fu Lei, Department of Anesthesiology, Qilu Hospital of Shandong University, 107 Wenhuan Xi Road, Jinan, Shandong 250012, P.R. China

E-mail: leiweifu001@163.com

Key words: gynecologic neoplasms, cell-mediated immunity, surgery, lidocaine, lymphocytes, apoptosis, proliferation serum at $48 \mathrm{~h}$ was significantly decreased following surgery in the control group, compared with the pre-surgical values (3.782 \pm 0.282 , vs. $4.089 \pm 0.339 \mathrm{pg} / \mathrm{ml}$, respectively) and the ratio of IFN- $\gamma$ to interleukin-4 was well preserved in the lidocaine group. In conclusion, the present study demonstrated that the intraoperative systemic administration of lidocaine exerted a protective effect on CMI in patients with cervical cancer undergoing radical hysterectomy. This may be beneficial in reducing the occurrence of postoperative septic complications and tumor metastasis formation.

\section{Introduction}

Postoperative immunosuppression is a generalized state in several types of surgery, which is involved in postoperative septic complications and tumor metastasis formation (1). To assess the changes in immune state underlying surgical stress, cell-mediated immunity (CMI) is often investigated (1-3). Although surgical procedures always cause an increase in the total number of circulating white blood cells, critical individual leukocyte subpopulations, including lymphocytes, are suppressed in number and function $(2,3)$. A decrease in the lymphocyte proliferation rate (1) and increase in apoptosis $(4,5)$ are the two predominant factors leading to the reduction in circulating lymphocyte numbers. In addition, surgery also shifts the balance of T-helper (Th)1/Th2 cells towards anti-CMI Th2 dominance, which is relevant to the immunosuppression of CMI (2).

Lidocaine is widely used as an analgesic and anti-hyperalgesic and exhibits antibacterial actions and beneficial effects on the inflammatory response (6-9). Previous studies have revealed that intraoperative systemic lidocaine benefits patients by reducing postoperative pain, analgesic consumption, postoperative nausea and vomiting, and even the duration spent in hospital (10-12). Data also demonstrates that lidocaine can protect rats from cecal ligation and puncture assault-associated septic organ failure $(7,9)$. However, whether lidocaine exerts a protective effect on CMI in patients undergoing surgery for the removal of a primary tumor remains to be elucidated. 
Peri-operative immune changes occur primarily as a result of surgical trauma and subsequent neuroendocrine responses, thus efforts to reduce the immunosuppressive effects of surgery require initiation in the pre-operative period (1). Therefore, in the present study, lidocaine was administered intravenously between the pre-anesthesia period and the point of discharge from the operating room. It was hypothesized that lidocaine may have a beneficial effect on CMI during the postoperative period in patients with cervical cancer undergoing radical hysterectomy.

\section{Patients and methods}

Patients. A total of 30 adult female patients aged between 25 and 65 years old, undergoing radical hysterectomy were recruited in the present prospective study between August 2013 and January 2014. Patients were excluded if they had a weight $<45 \mathrm{~kg}$ or $>65 \mathrm{~kg}$; a history of allergies to local anesthetics, bradycardia or heart block; severe respiratory, renal or hepatic disease, previous history of opioid medication use or a psychiatric medical history.

The study protocol was approved by the ethics committee of Qilu Hospital of Shandong University (Jinan, China) and performed according to the Declaration of Helsinki. Written informed consent was obtained from all participants prior to enrolment.

All participants were randomized into two groups, according to a computer-generated random number table, in which the patients received either intravenous lidocaine (Shanghai Zhi Pharma Co., Ltd., Shanghai, China) or normal saline (control group). The solutions were prepared in a $20 \mathrm{cc}$ syringe and labeled only with a case number by a nurse in a blinded-manner. The patients assigned to the lidocaine group received an intravenous bolus infusion of $1.5 \mathrm{mg} / \mathrm{kg}$ lidocaine $10 \mathrm{~min}$ prior to the induction of anesthesia, followed by continuous infusion at $1.5 \mathrm{mg} / \mathrm{kg} / \mathrm{h}$ using a Graseby 3100 syringe pump (Graseby Medical, Ltd., Watford, UK) until discharge from the operating room. The patients in the control group received the same volume of normal saline. All surgical procedures were performed by the same team of surgeons to avoid individual variability in operative techniques. The characteristics of the patients are listed in Table I.

Following the administration of $0.1 \mathrm{mg} / \mathrm{kg}$ midazolam (Jiangsu Nhwa Pharmaceutical Co., Ltd., Xuzhou, China), 1-2 mg/kg propofol (Fresenius Kabi AG, Bad Homburg vor der Höhe, Germany), $2 \mu \mathrm{g} / \mathrm{kg}$ fentanyl (Yichang Humanwell Pharmaceutical Co., Ltd., Yichang, China) and $0.6 \mathrm{mg} / \mathrm{kg}$ rocuronium (Taizhou Xianju Pharmaceutical Co., Ltd., Taizhou, China) intravenously, the patients were intubated and ventilated to maintain the end-tidal $\mathrm{CO}_{2}$ volume between 30 and $40 \mathrm{mmHg}$. Anesthesia was maintained using 1.5-3\% sevoflurane (Jiangsu Hengrui Medicine Co., Ltd., Lianyungang, China) in $1 \mathrm{l} / \mathrm{min} \mathrm{O}_{2}$. Fentanyl and rocuronium were added, according to heart rate or the bispectral index (S/5; GE Healthcare Life Sciences, Helsinki, Finland). Noninvasive arterial blood pressure, electrocardiography and pulse oximetry $(\mathrm{S} / 5)$ were monitored continuously. During surgery, the patients received intravenous infusion of lactated Ringer's solution (Shandong Hualu Pharmaceutical Co., Ltd., Muping, China) at a rate of $6-12 \mathrm{ml} / \mathrm{kg} / \mathrm{h}$.
Peripheral blood lymphocyte (PBL) isolation. A $10 \mathrm{ml}$ blood sample was drawn from the median cubital vein of each patient 1 day prior to surgery, following discharge from the operating room and at $48 \mathrm{~h}$ post-surgery. Serum was isolated at room temperature by centrifugation $(1,000 \mathrm{x} \mathrm{g}, 5 \mathrm{~min})$ and stored at $-20^{\circ} \mathrm{C}$ prior to assessment. Lymphocytes were isolated using Ficoll-paque density centrifugation (Haoyang Biological Manufacture Co., Ltd., Tianjin, China) at 1,000 x g for $15 \mathrm{~min}$ at $-20^{\circ} \mathrm{C}$. The cell viability was assayed by $0.4 \%$ trypan blue exclusion, which was $>95 \%$ in all cases. The collected cells were resuspended at a density of $1 \times 10^{6}$ cells $/ \mathrm{ml}$ in RPMI 1640 medium (Thermo Fisher Scientific, Shanghai, China) supplemented with $10 \%$ heat-inactivated fetal calf serum, and cultured at $37^{\circ} \mathrm{C}$ in a humidified $5 \% \mathrm{CO}_{2}$ atmosphere.

Cell proliferation. The suspended cells $\left(100 \mu \mathrm{l} ; 1 \times 10^{6} \mathrm{cells} / \mathrm{ml}\right)$ were cultivated with $5 \mu \mathrm{g} / \mathrm{ml}$ phytohemagglutinin (PHA) (Sigma-Aldrich, St. Louis, MO, USA) in a 96-well plate at $37^{\circ} \mathrm{C}$ and $5 \% \mathrm{CO}_{2}$ for $24 \mathrm{~h}$. RPMI 1640 was used as a blank control in each group. Subsequently, $10 \mu \mathrm{l}$ Cell Counting Kit-8 (CCK-8; Dojindo Molecular Technologies, Inc., Kumamoto, Japan) was added to each well $4 \mathrm{~h}$ prior to the end of stimulation, when the optical density values were measured at $450 \mathrm{~nm}$ (Model 722; INESA Instrument, Shanghai, China). Data are expressed as the mean \pm standard deviation of four wells.

Detection of early apoptosis using Annexin V-fluorescein isothiocyanate (FITC)/propidium iodide (PI) staining. Detection of early apoptosis in the PBLs was performed, according to the manufacturer's instructions, using an Annexin V FITC kit (Bogoo, Shanghai, China). In brief, the PBLs $\left(2 \times 10^{6}\right)$ were resuspended in $2 \mathrm{ml}$ ice-cold phosphate-buffered saline and washed twice. The collected PBLs were initially suspended in $400 \mu 11 \mathrm{X}$ binding buffer, following which $5 \mu \mathrm{l}$ Annexin V-FITC was added for $15 \mathrm{~min}$ in the dark followed by $10 \mu \mathrm{l}$ PI for 5 min in the dark. A negative control was used for each sample, in which the PBLs were incubatedwith binding buffer alone. For the positive control, the cells were incubated with Annexin V-FITC or PI alone. The cell sample was measured immediately using a flow cytometer.

Flow cytometry $(F C)$. The FC data were acquired within $24 \mathrm{~h}$ of staining using CellFit version 2.0 software and a FACSCalibur cytometer (BD Biosciences, San Jose, CA, USA). An argon ion laser excitation of $488 \mathrm{~nm}$ was used. The emitted light was detected by logarithmic amplification through barrier filters, which were specific for the emission range of the different fluorophores: 530/22 nm for FITC (fluorescence channel FL1) and 575/42 nm (FL2) for PI. The results of the lymphocyte typing and lymphocyte early apoptosis detection were obtained by quadrant analyses of FL1, vs. FL2 channel dot plots and presented as the percentage of gated lymphocytes.

ELISA assay. The level of high-mobility group protein B1 (HMGB1), interferon (IFN)- $\gamma$ and interleukin (IL)-4 in the serum were determined using ELISA kits (R\&D Systems, Inc., Minneapolis, MN, USA), according to the manufacturer's instructions. 
Table I. Demographic and clinical characteristics of the participants in the control and lidocaine groups.

\begin{tabular}{lccc}
\hline Characteristic & Control & Lidocaine & P-value \\
\hline ASA (n) & & & 11 \\
I & 10 & 4 & 1.00 \\
II & 5 & $44.2 \pm 11.8$ & 0.20 \\
Age (years) & $48.6 \pm 5.6$ & $155.8 \pm 9.7$ & 0.86 \\
Height (cm) & $156.4 \pm 8.9$ & $56.0 \pm 6.5$ & 0.11 \\
Weight (kg) & $56.9 \pm 7.6$ & $132.3 \pm 25.1$ & 0.74 \\
Duration of surgery (min) & $129.3 \pm 24.4$ & $152.3 \pm 14.1$ & 0.32 \\
Duration of anesthesia (min) & $158.0 \pm 16.9$ & & \\
\hline
\end{tabular}

Data are presented as the mean \pm standard deviation $(n=15)$. ASA, American Society of Anesthesiologists Physical Status classification.

Statistical analysis. SigmaPlot 12.5 (Systat Software, Inc., San Jose, CA, USA) was used for statistical analysis. The data distribution was evaluated using Levene's test. The normally distributed data are expressed as the mean \pm standard deviation and were compared using one-way analysis of variance or an unpaired t-test. Descriptive variables were subjected to $\chi^{2}$ analysis or Fisher's exact test, as appropriate. $\mathrm{P}<0.05$ was considered to indicate a statistically significant difference.

\section{Results}

Preoperative clinical parameters of patients. All 30 patients recruited in the present study completed the study. As shown in Table I, no significant differences were identified between the groups in terms of the American Society of Anesthesiologists class, age, height, weight, duration of surgery or anesthesia (13).

Lidocaine protects the proliferation of PBLs. The number of blood lymphocytes always falls peri-operatively, and decreases in the lymphocyte proliferation rate are considered to be one of its causes (1). Therefore, the present study initially examined the proliferation rate of PBLs using a CCK-8 assay. The results, as shown in Fig. 1, demonstrated that lidocaine improved the proliferation rate of the surgical stressor-induced lymphocytes. Therefore, lidocaine may exert a protective effect on lymphocyte function.

Lidocaine attenuates surgery-induced apoptosis of PBLs. Surgical stressors not only reduce lymphocyte proliferation, but they increase apoptosis of immune cells. To determine whether lidocaine exerted a protective effect on surgery-induced apoptosis of lymphocytes, the PBLs in the present study were stained with Annexin V-FITC/PI and assessed using flow cytometry. As shown in Fig. 2, intraoperative systemic lidocaine attenuated the surgery-induced apoptosis of the PBLs.

Lidocaine preserves levels of IFN- $\gamma$ and $I L-4$. The serum level of IFN- $\gamma$ at $48 \mathrm{~h}$ post-surgery in the control group was decreased significantly, compared with the pre-surgical level (3.782 \pm 0.282 , vs. $4.089 \pm 0.339, \mathrm{pg} / \mathrm{ml}$ respectively). However, no significant differences were observed in the levels of IFN- $\gamma$ in the lidocaine group or in the levels of IL- 4 in the control and lidocaine group, compared with the pre-surgical value. As

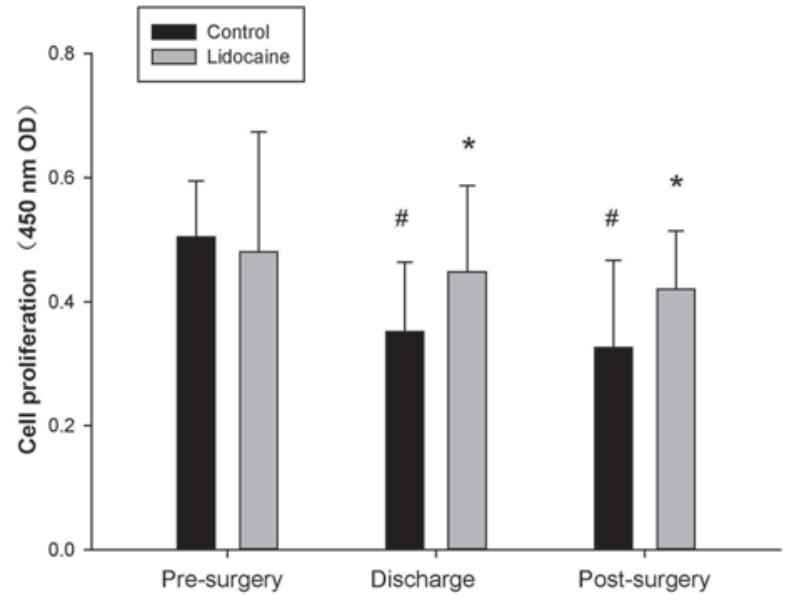

Figure 1. OD values of lymphocyte proliferation, detected using a Cell Counting kit- 8 assay. Data are presented as the mean \pm standard deviation $(\mathrm{n}=15)$. ${ }^{*} \mathrm{P}<0.05$, compared with the control; ${ }^{~} \mathrm{P}<0.05$, compared with pre-surgery. OD, optical density.

shown in Fig. 3, the ratio of IFN- $\gamma$ to IL-4 was well preserved in the lidocaine group.

Lidocaine decreases the protein levels of HMGB1 in the serum. HMGB1, as a critical mediator of several inflammatory and non-inflammatory diseases, is important in surgery-associated sepsis and tumor metastasis. To assess whether lidocaine has an inhibitory effect on its expression in patients undergoing surgery, the serum level of HMGB1 was determined in the present study using ELISA. As expected, the serum protein level of HMGB1 in the lidocaine group was reduced, which was significantly difference 48 h-post surgery, compared with the control group (Fig. 4).

\section{Discussion}

Surgery-induced stress can increase the susceptibility to tumor metastasis $(14,15)$, and it is considered to be one of the causes of the suppression of CMI secondary to surgical stress (1). The present study indicated that peri-operative intravenous lidocaine had a beneficial effect on CMI, and this was associated with the preservation of lymphocyte proliferation, attenuation 


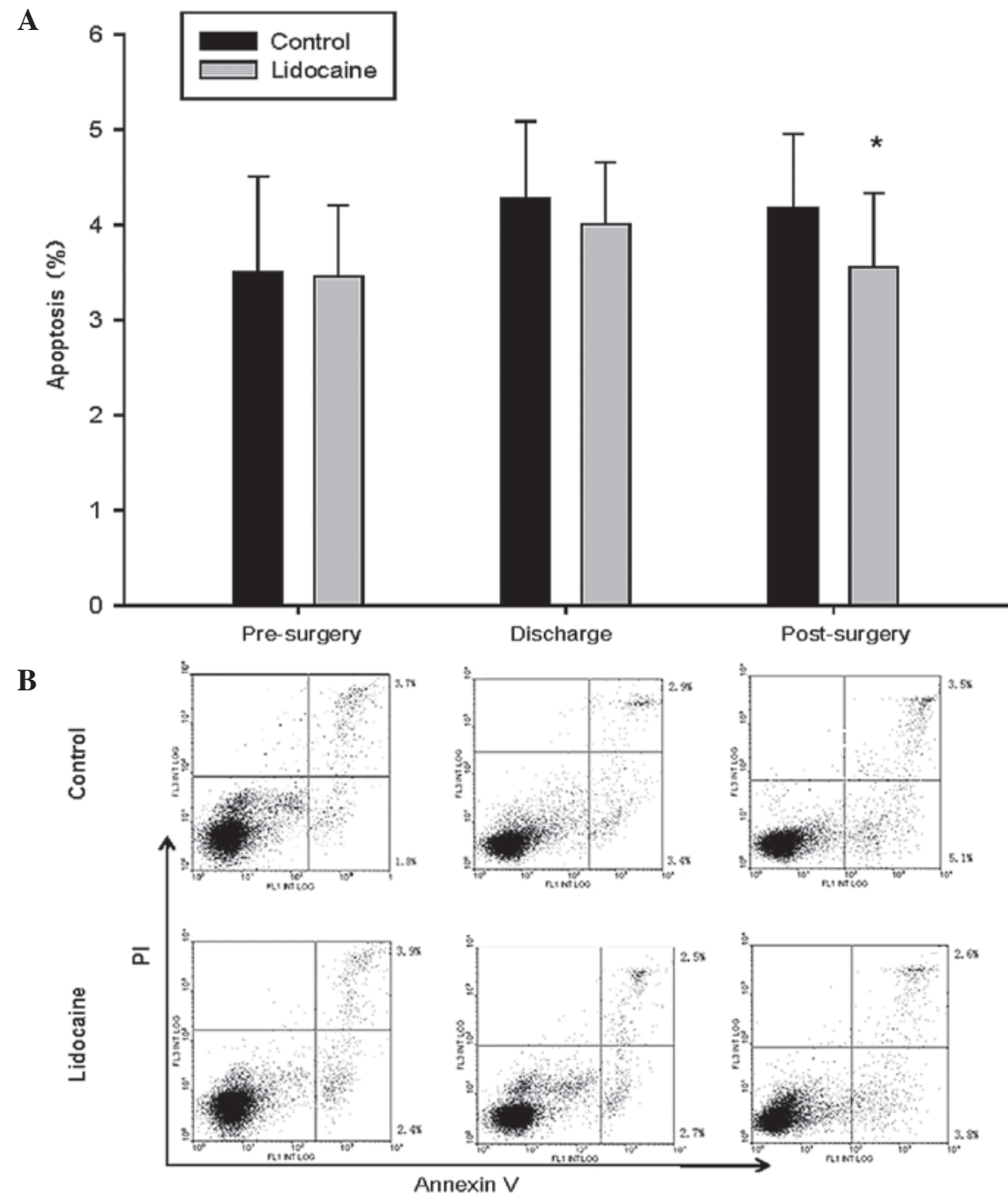

Figure 2. Apoptosis and proliferation of PBLs. (A) Percentage of PBLs that have undergone apoptosis and (B) representative dot-blot following fluorescein isocyothianate-Annexin V and PI staining. Data are presented as the mean values \pm standard deviation. ( $\mathrm{n}=15)$. * $\mathrm{P}<0.05$, compared with the control. PBLs, peripheral blood lymphocytes; PI, propidium iodide.

of apoptosis, maintainence of the balance of Th1/Th2 cells and the decreased production of cytokines in patients undergoing radical hysterectomy.

Lymphocytes are the major cellular components of the immune response and are important in the recovery of patients following surgery for the removal of a tumor (16). However, the decrease in lymphocyte proliferation rate (1) and the increase in apoptosis $(4,5)$ are common following surgery and are considered to be the two predominant factors leading to the decrease of lymphocytes in the circulation. The lymphocyte mitogenic response to PHA is an important tool, which can mimic the activity of lymphocytes in response to an exogenous stimulus, and was well-preserved in the patients in the lidocaine group, compared with those in the control group in the present study. This result is similar to that of Yardeni et al (10). In addition, the results of the present study revealed that intraoperative systemic lidocaine attenuated the apoptosis of PBL cells induced by surgical stressors. However, previous in vitro studies have demonstrated the opposite conclusions regarding cell proliferation $(17,18)$ and apoptosis $(19,20)$. This indicates that the protective effects of lidocaine on $\mathrm{CMI}$ in vivo are not directly due to its effects on lymphocytes, and the underlying mechanisms require further exploration.

Distinct patterns of cytokines are produced by two types of Th cell. Th1 lymphocytes produce cytokines, including IFN- $\gamma$ and IL-2, which favor CMI; whereas Th2 cells secrete cytokines, including IL-4 and IL-10, which favor humoral immunity (21). Itis well-established that surgery reduces the number of Th1 cytokines, including IFN- $\gamma$, and decreases the Th1/Th2 ratio, which is responsible for the suppression of CMI following surgery (21). This was observed in the patients in the control group in the present study, however, the serum levels of IFN- $\gamma$ did not change significantly in the patients in the lidocaine group, which maintained the balance of Th1/Th2.

HMGB1, as a critical mediator of several inflammatory diseases, is important in surgery-associated sepsis (22). It is also implicated in non-inflammatory conditions, including cancer, by regulating tumorigenesis and contributing to metastasis (23). HMGB1 levels increase significantly and last for a few days following liver resection (24) or cardiac surgery (25). In the present study, although the increased level of HMGB1 was not significant following surgery compared with the pre-surgical levels in the control group, there was a significant 
A

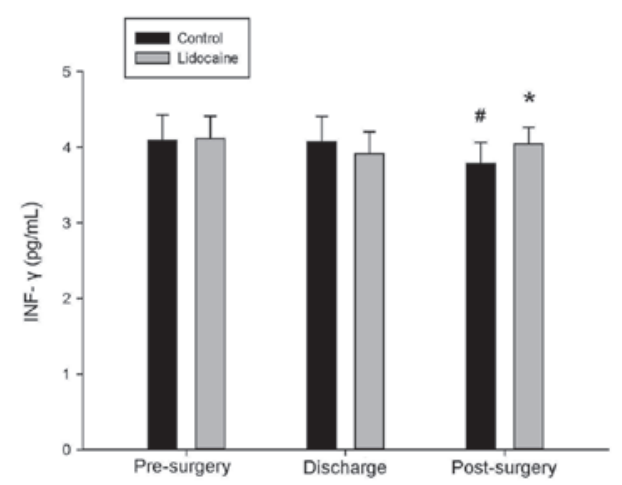

B

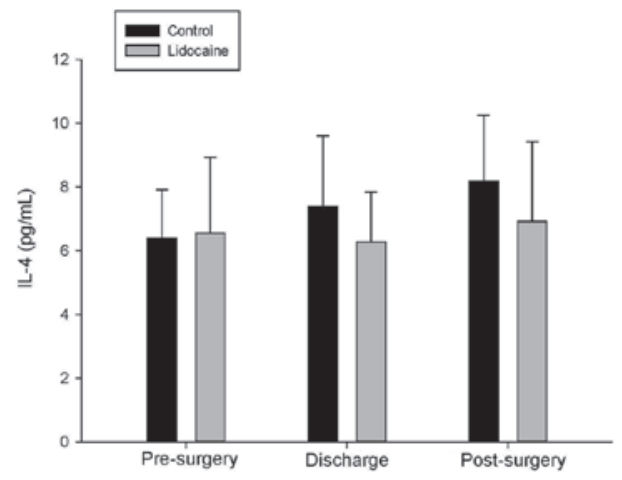

C

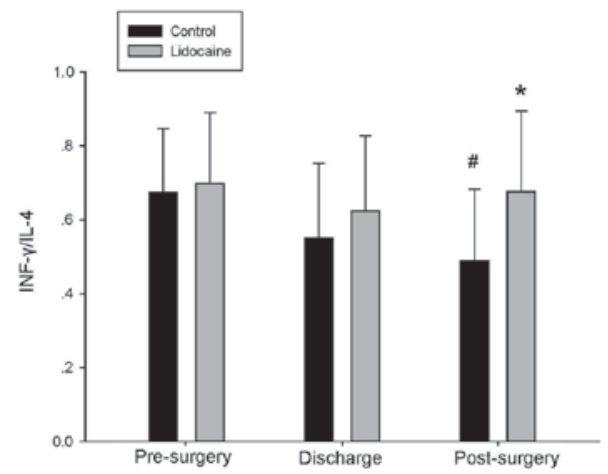

Figure 3. Expression of cytokines. (A) Serum levels of IFN- $\gamma$, (B) IL-4 and (C) the ratio of IFN- $\gamma$ to IL-4. Data are presented as the mean \pm standard deviation ( $\mathrm{n}=15)$. " $\mathrm{P}<0.05$, compared with the control; ${ }^{\mathrm{P}} \mathrm{P}<0.05$, compared with pre-surgery. IFN, interferon; IL, interleukin.

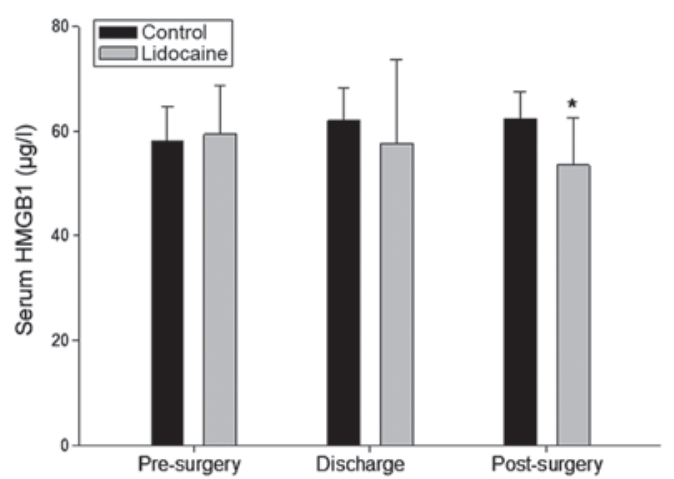

Figure 4. Serum protein levels of HMGB1 in patients undergoing radical hysterectomy. Data are presented as the mean \pm standard deviation $(n=15)$. ${ }^{*} \mathrm{P}<0.05$, vs. control. HMGB1, high-mobility group protein $\mathrm{B} 1$. decrease at $48 \mathrm{~h}$ post-surgery in the lidocaine group, compared with the control group. It was hypothesized that lidocaine, used as an anesthetic adjuvant decreases the level of HMGB1 and may contribute to attenuate the complications of tumor surgery, including sepsis and metastasis.

There are certain limitations to the present study. Previous studies have revealed that lidocaine decreases plasma concentrations of cortisol (26) and catecholamines (27) in patients undergoing surgery, whereas glucocorticoids reduce $\mathrm{T}$ cell proliferation and increase the apoptosis of immature $\mathrm{T}$ cells (16). Catecholamines suppress anti-metastatic immunity and act directly on minimal residual disease to promote metastatic progression (16). However, the present study did not further examine the mechanism underlying the protective effect of lidocaine on CMI. Secondly, investigations are required to determine the direct association between suppressed CMI and tumor metastases in patients undergoing surgical removal of a primary tumor.

In conclusion, the present study demonstrated that the intraoperative systemic administration of lidocaine exerted a protective effect on $\mathrm{CMI}$ in patients with cervical cancer undergoing radical hysterectomy. This may be beneficial in reducing the occurrence of postoperative septic complications and tumor metastasis formation.

\section{Acknowledgements}

This study was supported by the Qilu Hospital Science Research Foundation (grant no. 26010175616032), and in part, by Shandong Provincial Natural Science Foundation, China (grant nos. Y2007C115, ZR2011HM028 and 2009ZRB14031).

\section{References}

1. Hogan BV, Peter MB, Shenoy HG, Horgan K and Hughes TA: Surgery induced immunosuppression. Surgeon 9: 38-43, 2011.

2. Ogawa K, Hirai M, Katsube T, Murayama M, Hamaguchi K, Shimakawa T, Naritake Y, Hosokawa T and Kajiwara T: Suppression of cellular immunity by surgical stress. Surgery 127: 329-336, 2000

3. Ishikawa M, Nishioka M, Hanaki N, Miyauchi T, Kashiwagi Y, Ioki H, Kagawa A and Nakamura Y: Perioperative immune responses in cancer patients undergoing digestive surgeries. World J Surg Oncol 7: 7, 2009.

4. Delogu G, Moretti S, Antonucci A, Marcellini S, Masciangelo R, Famularo G, Signore L and De Simone C: Apoptosis and surgical trauma: Dysregulated expression of death and survival factors on peripheral lymphocytes. Arch Surg 135: 1141-1147, 2000.

5. Papadima A, Boutsikou M, Lagoudianakis EE, Kataki A, Konstadoulakis M, Georgiou L, Katergiannakis V and Manouras A: Lymphocyte apoptosis after major abdominal surgery is not influenced by anesthetic technique: A comparative study of general anesthesia versus combined general and epidural analgesia. J Clin Anesth 21: 414-421, 2009.

6. Hollmann MW and Durieux ME: Local anesthetics and the inflammatory response: A new therapeutic indication? Anesthesiology 93: 858-875, 2000.

7. Gallos G, Jones DR, Nasr SH, Emala CW and Lee HT: Local anesthetics reduce mortality and protect against renal and hepatic dysfunction in murine septic peritonitis. Anesthesiology 101: 902-911, 2004.

8. Wang HL, Zhang WH, Lei WF, Zhou CQ and Ye T: The inhibitory effect of lidocaine on the release of high mobility group box 1 in lipopolysaccharide-stimulated macrophages. Anesth Analg 112: 839-844, 2011.

9. Wang HL, Xing YQ, Xu YX, Rong F, Lei WF and Zhang WH: The protective effect of lidocaine on septic rats via the inhibition of high mobility group box 1 expression and NF- $\mathrm{BB}$ activation. Mediators Inflamm 2013: 570370, 2013. 
10. Yardeni IZ, Beilin B, Mayburd E, Levinson Y and Bessler H: The effect of perioperative intravenous lidocaine on postoperative pain and immune function. Anesth Analg 109: 1464-1469, 2009.

11. Farag E, Ghobrial M, Sessler DI, Dalton JE, Liu J, Lee JH, Zaky S, Benzel E, Bingaman W and Kurz A: Effect of perioperative intravenous lidocaine administration on pain, opioid consumption and quality of life after complex spine surgery. Anesthesiology 119: 932-940, 2013.

12. Yon JH, Choi GJ, Kang H, Park JM and Yang HS: Intraoperative systemic lidocaine for pre-emptive analgesics in subtotal gastrectomy: A prospective, randomized, double-blind, placebo-controlled study. Can J Surg 57: 175-182, 2014.

13. Fitz-Henry J: The ASA classification and peri-operative risk. Ann R Coll Surg Engl 93: 185-187, 2011.

14. Goldfarb Y, Sorski L, Benish M, Levi B, Melamed R and Ben-Eliyahu S: Improving postoperative immune status and resistance to cancer metastasis: A combined perioperative approach of immunostimulation and prevention of excessive surgical stress responses. Ann Surg 253: 798-810, 2011.

15. Glasner A, Avraham R, Rosenne E, Benish M,Zmora O, Shemer S, Meiboom $\mathrm{H}$ and Ben-Eliyahu S: Improving survival rates in two models of spontaneous postoperative metastasis in mice by combined administration of a beta-adrenergic antagonist and a cyclooxygenase-2 inhibitor. J Immunol 184: 2449-2457, 2010.

16. Neeman E and Ben-Eliyahu S: Surgery and stress promote cancer metastasis: New outlooks on perioperative mediating mechanisms and immune involvement. Brain Behav Immun 30 (Suppl): S32-S40, 2013

17. Lahat A,Ben-Horin S,Lang A, Fudim E, Picard O and Chowers Y: Lidocaine down-regulates nuclear factor-kappaB signalling and inhibits cytokine production and T cell proliferation. Clin Exp Immunol 152: 320-327, 2008.

18. Kawasaki T, Kawasaki C, Sata T and Chaudry IH: Lidocaine suppresses mouse Peyer's Patch T cell functions and induces bacterial translocation. Surgery 149: 106-113, 2011.
19. Werdehausen R, Braun S, Essmann F, Schulze-Osthoff K, Walczak H, Lipfert P and Stevens MF: Lidocaine induces apoptosis via the mitochondrial pathway independently of death receptor signaling. Anesthesiology 107: 136-143, 2007.

20. Boselli E, Duflo F, Debon R, Allaouchiche B, Chassard D, Thomas L and Portoukalian J: The induction of apoptosis by local anesthetics: A comparison between lidocaine and ropivacaine. Anesth Analg 96: 755-756, 2003.

21. Decker D, Schondorf M, Bidlingmaier F, Hirner A and von Ruecker AA: Surgical stress induces a shift in the type-1/type-2 T-helper cell balance, suggesting down-regulation of cell-mediated and up-regulation of antibody-mediated immunity commensurate to the trauma. Surgery 119: 316-325, 1996.

22. Wang H, Ward MF and Sama AE: Targeting HMGB1 in the treatment of sepsis. Expert Opin Ther Targets 18: 257-268, 2014.

23. Tang D, Kang R, Zeh HJ III and Lotze MT: High-mobility group box 1 and cancer. Biochim Biophys Acta 1799: 131-140, 2010.

24. Tsujii S, Okabayashi T, Shiga M, Takezaki Y, Sugimoto T, Kobayashi M and Hanazaki K: The effect of the neutrophil elastase inhibitor sivelestat on early injury after liver resection. World J Surg 36: 1122-1127, 2012.

25. Hasegawa A, Iwasaka H, Hagiwara S, Koga H, Hasegawa R, Kudo K, Kusaka J and Noguchi T: Anti-inflammatory effects of perioperative intensive insulin therapy during cardiac surgery with cardiopulmonary bypass. Surg Today 41: 1385-1390, 2011.

26. El-Tahan MR, Warda OM, Diab DG, Ramzy EA and Matter MK: A randomized study of the effects of perioperative i.v. lidocaine on hemodynamic and hormonal responses for cesarean section. J Anesth 23: 215-221, 2009.

27. Palacios R and Sugawara I: Hydrocortisone abrogates proliferation of $\mathrm{T}$ cells in autologous mixed lymphocyte reaction by rendering the interleukin-2 Producer T cells unresponsive to interleukin-1 and unable to synthesize the T-cell growth factor. Scand J Immunol 15: 25-31, 1982. 